\title{
Wigner-Dyson statistics from the replica method
}

\author{
Alex Kamenev†† and Marc Mézard†§ \\ $\dagger$ Institute for Theoretical Physics, University of California Santa Barbara, CA 93106-4030, USA \\ ¥ Department of Physics, University of California Santa Barbara, CA 93106-4030, USA \\ $\S$ CNRS, Laboratoire de Physique Théorique de l'ENS, 24 rue Lhomond, 75231 Paris Cedex 05, \\ France
}

Received 14 January 1999

\begin{abstract}
We compute the correlation functions of the eigenvalues in the Gaussian unitary ensemble using the fermionic replica method. We show that non-trivial saddle points, which break replica symmetry, must be included in the calculation in order to correctly reproduce the exact asymptotic behaviour of the correlation functions at a large distance.
\end{abstract}

\section{Introduction}

Random matrix theory (RMT) has found broad applications in physics ranging from nuclear spectra to electrons in metallic grains, see $[1,2]$ for reviews. The first mathematically rigorous results for the level statistics were derived by Gaudin [3] and Dyson [4] using the method of orthogonal polynomials. The progress in the theory of Anderson localization in the late 1970s established a close relation between the RMT and matrix $\sigma$-models initially in their replicated form [5,6]. Despite of a lot of efforts [7, 8], however, the initial attempts to reproduce Dyson's results from the replicated $\sigma$-models were not successful: while the density of eigenvalues could be found easily, it seemed that the eigenvalue correlations could not be obtained from the replicated $\sigma$-models. The most detailed account of such attempts was probably given by Zirnbauer and Verbaarschot [8], who computed both bosonic and fermionic replicated $\sigma$-models and obtained different results, both differing from the correct one. Only the supersymmetric (SUSY) formulation of the $\sigma$-model introduced by Efetov [10] gave a correct and beautiful way of calculating the correlations of eigenvalues from a $\sigma$-model formulation. It has thus become common knowledge over the last 15 years that the SUSY is the only field theoretic method capable of computing the RMT level statistics, while the internal subtleties of the replica method seem to make it inapplicable for this task.

Looking at the replicated $\sigma$-model approach, it is clear that there is one underlying assumption in the existing computations, which is the absence of any spontaneous breaking of replica symmetry. The study of the statistics of eigenvalues of a large $N \times N$ random matrix is mapped exactly onto a $\sigma$-model, where the action is of order $N$. Among various saddle points of the $\sigma$-model which could a priori contribute, only the trivial 'replica symmetric' one was discussed. In this paper we revisit the problem, and consider all possible saddle points. Because of the symmetry of the $\sigma$-model the saddle points are actually saddle point manifolds. We show that the computation of the $r$-point correlation function involves $2^{r}$ saddle point manifolds. For the one-point function (the level density), the trivial saddle point gives Wigner's semicircle law, whereas the second one contributes to order $1 / N$, and is, in fact, 
needed to obtain the oscillatory component of the density of states (DOS). For the two-point function, the effect is even more dramatic since one of the extra three non-trivial saddle point manifolds contributes to the leading order at large $N$ (the other two being $1 / N$ corrections). Taking it into account gives the correct result for the two-point correlation function. A similar situation was actually found in the SUSY, where Andreev and Altshuler [11] showed that the asymptotic behaviour of the two-level correlations at large energy differences (in units of the mean level spacing) can be obtained by the saddle point evaluation of the SUSY $\sigma$-model, including one extra non-trivial saddle point.

Here we restrict ourselves to the Gaussian unitary ensemble (GUE), where we derive the exact results for the DOS, including oscillatory $1 / N$ correction as well as the large energy difference behaviour of the two-point correlation function using the fermionic replica formalism. The strategy is simple enough to be easily generalized for the higher correlation functions for which it also leads to known GUE expressions. Technically, a very useful step is to integrate exactly over the angular degrees of freedom of the $\sigma$-model, using the ItzyksonZuber integral [12], which leaves the $n$ eigenvalues of the replicated $\sigma$-model as the only integration variables. The saddle points are thus discussed on the level of eigenvalues, and the resulting replica symmetry breaking (RSB) appears to be a particularly simple version of the vector RSB mechanism encountered in several disordered systems [13].

The paper is organized as follows: in section 2 we present the calculations of the average DOS, including the $1 / N$ corrections. This section is also used to introduce notations and illustrate the technique on this simple, but instructive example. Calculation of the two-point correlation function is given in section 3. Finally, sections 4 and 5 are devoted to discussions of the method and remaining open questions. Some technical details are presented in the three appendices.

\section{Density of states}

We are interested in the spectral properties of the random $N \times N$ Hermitian Hamiltonians, $H$, with the Gaussian probability distribution function

$$
P(H)=2^{N(N-1) / 2}\left(\frac{N}{2 \pi}\right)^{N^{2} / 2} \exp \left\{-\frac{N}{2} \operatorname{Tr} H^{2}\right\} .
$$

We begin with the calculation of the one-point function $S_{1}(E)$ defined as

$$
S_{1}(E)=N^{-1} \overline{\operatorname{Tr}(E-H)^{-1}}
$$

where the complex energy $E$ has an infinitesimal negative imaginary part. The bar stands for the averaging over $H$ with the measure given by equation (1). The DOS is given by the imaginary part of this correlation function

$$
\nu(E)=\pi^{-1} \operatorname{Im} S_{1}(E) .
$$

Introducing $2 N$ anti-commuting variables $\psi_{x}, \bar{\psi}_{x}$, where $x$ is a discrete index $x \in\{1, \ldots, N\}$, the correlation function may be written as

$$
S_{1}(E)=N^{-1} \overline{\frac{\partial}{\partial E} \ln Z(E)}
$$

where

$$
Z(E)=\int \prod_{x=1}^{N} \mathrm{~d} \bar{\psi}_{x} \mathrm{~d} \psi_{x} \exp \left\{-\sum x, y=1^{N} \bar{\psi}_{x}\left(E \delta_{x y}-H_{x y}\right) \psi_{y}\right\}
$$


We use now the replica trick to write the logarithm as $\ln Z=\lim _{n \rightarrow 0}\left(Z^{n}-1\right) / n$. As a result the correlation function takes the form

$$
S_{1}(E)=\lim _{n \rightarrow 0} \frac{1}{n} S_{1}^{(n)}(E) \quad S_{1}^{(n)}(E)=N^{-1} \frac{\partial}{\partial E} \overline{Z(E)^{n}} .
$$

It is convenient to introduce the generating function $Z^{(n)}(\hat{E}) \equiv Z\left(E_{1}\right) \ldots Z\left(E_{n}\right)$, where $\hat{E}$ is a diagonal $n \times n$ matrix which has the form $\hat{E}=\operatorname{diag}\left\{E_{1}, \ldots, E_{n}\right\}$. In the limit $E_{1}, \ldots, E_{n} \rightarrow E$ the generating function $Z^{(n)}(\hat{E})$ approaches $Z(E)^{n}$ and gives the one-point function according to equation (6). The generating function $Z^{(n)}(\hat{E})$ may be written as the fermionic integral analogous to that of equation (5). The integration runs now over $2 n N$ fermionic variables $\psi_{x}^{j}, \bar{\psi}_{x}^{j}$, where $x \in\{1, \ldots, N\}$ and $j \in\{1, \ldots, n\}$. Performing the averaging over the random matrix $H$ one finds

$$
\overline{Z^{(n)}(\hat{E})}=\int \prod_{x=1}^{N} \prod_{j=1}^{n} \mathrm{~d} \bar{\psi}_{x}^{j} \mathrm{~d} \psi_{x}^{j} \exp \left\{-\sum_{x, j} \bar{\psi}_{x}^{j} E_{j} \psi_{x}^{j}-\frac{1}{2 N} \sum_{j, k=1}^{n} T_{j k} T_{k j}\right\}
$$

where $T_{j k} \equiv \sum_{x} \bar{\psi}_{x}^{j} \psi_{x}^{k}$. Introducing the Hubbard-Stratonovich decoupling of the last term with the $x$-independent, Hermitian $n \times n$ matrix $\hat{Q}$ and integrating out the anti-commuting variables, one obtains the $\sigma$-model in the form $[5,6]$ :

$$
\begin{aligned}
& \overline{Z^{(n)}(\hat{E})}=c_{n} \int \mathrm{d}[\hat{Q}] \exp \left\{-\frac{N}{2} \operatorname{Tr} \hat{Q}^{2}+N \operatorname{Tr} \ln (\hat{E}-\mathrm{i} \hat{Q})\right\} \\
& c_{n}=\left(\frac{N}{2 \pi}\right)^{n^{2} / 2} 2^{n(n-1) / 2} .
\end{aligned}
$$

The standard route to study the large $N$ limit of the DOS prescribes to take all energies equal to $E$, look for the saddle points of the functional integral, equation (8), and then consider fluctuations around the saddle points (see e.g. [8]). The saddle point equation, $\hat{Q}=(\hat{Q}+\mathrm{i} E)^{-1}$, may be solved by going to the basis where $\hat{Q}$ is diagonal. One finds two possible solutions for each of the eigenvalues $\lambda_{1}, \ldots, \lambda_{n}$ of $\hat{Q}+\mathrm{i} E$ :

$$
\lambda_{ \pm}(E)= \pm \sqrt{1-\frac{E^{2}}{4}}+\frac{\mathrm{i} E}{2} .
$$

This results in $2^{n}$ distinct diagonal matrices. As $\hat{Q}$ is obtained from the diagonal matrix through a unitary transformation, one finds $n+1$ saddle point manifolds, generated by rotations of $U(n) /[U(p) U(n-p)]$, applied to a diagonal matrix with $\lambda_{i}=\lambda_{-}$for $i=1 \ldots p$ and $\lambda_{j}=\lambda_{+}$for $j=p+1 \ldots n$, where $0 \leqslant p \leqslant n$. Which of these manifolds dominates is not obvious a priori $\uparrow$. It is known that the leading, large $N$, contribution to the DOS is given by the simple 'replica symmetric' saddle point (this one is just a point not a manifold, since $\hat{Q}$ is proportional to 11 ) with $p=0$ [8]. We show below that other saddle point manifolds are crucial for computations of the $1 / N$ corrections to the DOS, as well as the level correlations. In order to take into account all possible saddle points, one must be careful to integrate properly over the manifolds. To achieve this we found it convenient to employ the method introduced by Guhr [14] in the SUSY context. This method takes advantage of the Itzykson-Zuber integral [12] for the GUE to integrate all rotational degrees of freedom exactly, and discusses the

$\dagger$ In the limit where the imaginary part of $E$ vanishes, the actions of the various saddle points differ only by phases. If one is interested only in the DOS to leading order at large $N$, one may choose the imaginary part of the energy, $\eta$, to be such that $\eta N \gg 1$. This makes the trivial saddle point with $p=0$ dominant over all others. However, this procedure, which smoothes the positions of individual levels, is not allowed if one computes oscillatory parts of correlation functions. 
saddle points in terms of the eigenvalues of $\hat{Q}$. A similar method has been employed by Brézin and Hikami in order to derive and extend the universality of level spacing distributions [15].

By first shifting the $\hat{Q}$ matrix $\hat{Q} \rightarrow \hat{Q}-\mathrm{i} \hat{E} \dagger$ one obtains:

$$
\overline{Z^{(n)}(\hat{E})}=c_{n}(-\mathrm{i})^{N n} \int \mathrm{d}[\hat{Q}] \exp \left\{-\frac{N}{2} \operatorname{Tr}(\hat{Q}-\mathrm{i} \hat{E})^{2}\right\}(\operatorname{det} \hat{Q})^{N} .
$$

The Hermitian matrix $\hat{Q}$ is then diagonalized by a unitary transformation, $\hat{Q}=U^{-1} \hat{\Lambda} U$ with $U \in U(n)$ and the diagonal matrix $\hat{\Lambda}=\operatorname{diag}\left\{\lambda_{1}, \ldots, \lambda_{n}\right\}$. The volume element transforms like

$$
\begin{aligned}
\mathrm{d}[\hat{Q}] & =\Delta_{n}^{2}(\hat{\Lambda}) \mathrm{d}[\hat{\Lambda}] \mathrm{d} \mu(U) \\
\mathrm{d}[\hat{\Lambda}] & =\prod_{j=1}^{n} d \lambda_{j}
\end{aligned}
$$

where $\mathrm{d} \mu(U)$ is the measure of the group $U(n)$ and the Jacobian is given by the square of the Vandermonde determinant

$$
\Delta_{n}(\hat{\Lambda}) \equiv \prod_{1 \leqslant i<j \leqslant n}\left(\lambda_{j}-\lambda_{i}\right) .
$$

The non-trivial integration over the group $U(n)$ in equation (11) may be performed using the Itzykson-Zuber integral [12] (see also appendix 5 of [1]):

$$
\begin{aligned}
& \overline{Z^{(n)}(\hat{E})}= c_{n}(-\mathrm{i})^{N n} \int \mathrm{d}[\hat{\Lambda}] \Delta_{n}^{2}(\hat{\Lambda})(\operatorname{det} \hat{\Lambda})^{N} \int \mathrm{d} \mu(U) \exp \left\{-\frac{N}{2} \operatorname{Tr}\left(\hat{\Lambda}-\mathrm{i} U \hat{E} U^{-1}\right)^{2}\right\} \\
&= c_{n}(-\mathrm{i})^{N n}\left(\frac{\pi}{N}\right)^{n(n-1) / 2} \frac{1}{\Delta_{n}(\mathrm{i} \hat{E})} \int \mathrm{d}[\hat{\Lambda}] \Delta_{n}(\hat{\Lambda})(\operatorname{det} \hat{\Lambda})^{N} \exp \left\{-\frac{N}{2} \sum_{j=1}^{n}\left(\lambda_{j}\right.\right. \\
&\left.\left.-\mathrm{i} E_{j}\right)^{2}\right\} .
\end{aligned}
$$

It may seem that the last expression has poles at $E_{i}=E_{j}$, which is not the case. Indeed, the integral over the eigenvalues $\lambda_{j}$ results in a totally antisymmetric function of $E_{i}$, which vanishes if $E_{i}=E_{j}$. The reason we introduced the diagonal matrix $\hat{E}$ with all elements different was to regularize properly this fictitious singularity. The next step is to take the limit $E_{1}, \ldots, E_{n} \rightarrow E$. This procedure is described in appendix A. The result is given by

$$
\overline{Z^{n}(E)}=c_{n}^{\prime} \int \mathrm{d}[\hat{\Lambda}] \Delta_{n}^{2}(\hat{\Lambda}) \exp \left\{-N \sum_{j=1}^{n} A\left(\lambda_{j}, E\right)\right\}
$$

where

$$
A\left(\lambda_{j}, E\right)=\frac{1}{2}\left(\lambda_{j}-\mathrm{i} E\right)^{2}-\ln \lambda_{j} \quad c_{n}^{\prime}=(-\mathrm{i})^{N n} \frac{N^{n^{2} / 2}}{(2 \pi)^{n / 2}} \frac{1}{\prod_{j=1}^{n} j !} .
$$

Equation (15) may be easily derived already from the first line of equation (14) if $\hat{E}$ is proportional to the unit matrix. The reason the longer procedure was presented is to generalize it later for the case of multi-point correlation functions. Employing equation (6), one finally obtains for the replicated single-point correlation function

$S_{1}^{(n)}(E)=\mathrm{i} c_{n}^{\prime} \int \mathrm{d}[\hat{\Lambda}] \Delta_{n}^{2}(\hat{\Lambda}) \exp \left\{-N \sum_{j=1}^{n} A\left(\lambda_{j}, E\right)\right\}\left[\sum_{j=1}^{n}\left(\lambda_{j}-\mathrm{i} E\right)\right]$.

$\dagger$ To preserve the Hermiticity one may choose the elements of the $\hat{E}$ matrix on the negative imaginary axis and perform the analytical continuation to the real axis after the group integration. Since the resulting expression is an analytic function of all $E_{i}$ in the lower half plane, this procedure is legitimate. 
So far all the calculations have been exact. One may now take advantage of the large parameter $N \gg 1$ to perform the integrations in equation (17) by the saddle point method. Differentiating $A\left(\lambda_{j}, E\right)$ over $\lambda_{j}$, one finds two saddle point solutions for each $\lambda_{j}$, which are given by $\lambda_{ \pm}(E)$ defined by equation (10). This leads to $2^{n}$ distinct saddle points of the integral in equation (17), each of them may be brought to the form

$$
\hat{\Lambda}=\operatorname{diag}\{\underbrace{\lambda_{-}, \ldots, \lambda_{-}}_{p}, \underbrace{\lambda_{+}, \ldots, \lambda_{+}}_{n-p}\}
$$

There are $C_{n}^{p}=n ! /[p !(n-p) !]$ such saddle points for every $p, 0 \leqslant p \leqslant n$. Finding dominant saddle points is not totally trivial. On the one hand, the saddle point action, $A_{ \pm}=A\left(\lambda_{ \pm}(E), E\right)$, is such that $\left|A_{+}\right|=\left|A_{-}\right|$when the energy is real (see footnote on page 3$)$. Furthermore, one must be careful with the saddle point calculation of the integral in equation (17) because the pre-exponential factor, $\Delta_{n}^{2}(\hat{\Lambda})$, vanishes identically at any saddle point (for $n>2$ ). Therefore, it should be expanded to a sufficiently high power of $\lambda_{j}-\lambda_{ \pm}$ to produce a non-zero result. To this end we introduce variables $\xi_{j}$ describing fluctuations around the saddle point, equation (18), defined as:

$$
\begin{array}{ll}
\lambda_{i}=\lambda_{-}+\xi_{i} / \sqrt{N} & i=1 \ldots p \\
\lambda_{j}=\lambda_{+}+\xi_{j} / \sqrt{N} & j=p+1 \ldots n
\end{array}
$$

and the diagonal matrices $\hat{\Xi}_{-}=\operatorname{diag}\left\{\xi_{1} \ldots \xi_{p}\right\}$ and $\hat{\Xi}_{+}=\operatorname{diag}\left\{\xi_{p+1} \ldots \xi_{n}\right\}$. For any $p$ one may identically rewrite $\Delta_{n}^{2}(\hat{\Lambda})$ as

$$
\begin{aligned}
\Delta_{n}^{2}(\hat{\Lambda})=\left(\frac{1}{\sqrt{N}}\right)^{p(p-1)+(n-p)(n-p-1)}\left[\prod_{i=1}^{p} \prod_{j=p+1}^{n}\left(\lambda_{+}-\lambda_{-}+\frac{\xi_{j}-\xi_{i}}{\sqrt{N}}\right)\right]^{2} \\
\quad \times \Delta_{p}^{2}\left(\hat{\Xi}_{-}\right) \Delta_{n-p}^{2}\left(\hat{\Xi}_{+}\right) .
\end{aligned}
$$

The factor in square brackets on the rhs of this expression is non-vanishing at the saddle point and therefore may be substituted by its saddle point value. Expanding the exponent to second order in the deviations from the saddle point, one obtains for the replicated correlation function

$$
\begin{aligned}
S_{1}^{(n)}(E)=\mathrm{i} c_{n}^{\prime} & \sum_{p=0}^{n} C_{n}^{p}\left[\lambda_{+}-\lambda_{-}\right]^{2 p(n-p)} \mathrm{e}^{-N p A_{-}-N(n-p) A_{+}}\left[p \lambda_{-}+(n-p) \lambda_{+}-\mathrm{i} n E\right] \\
& \times\left(\frac{1}{\sqrt{N}}\right)^{p^{2}+(n-p)^{2}} \int \prod_{i=1}^{p} \mathrm{~d} \xi_{i} \Delta_{p}^{2}\left(\hat{\Xi}_{-}\right) \exp \left\{-\frac{1}{2} A_{-}^{\prime \prime} \sum_{i=1}^{p} \xi_{i}^{2}\right\} \\
& \times \int \prod_{j=p+1}^{n} \mathrm{~d} \xi_{j} \Delta_{n-p}^{2}\left(\hat{\Xi}_{+}\right) \exp \left\{-\frac{1}{2} A_{+}^{\prime \prime} \sum_{j=p+1}^{n} \xi_{j}^{2}\right\}
\end{aligned}
$$

where $A_{ \pm}^{\prime \prime}=\left.\partial_{\lambda}^{2} A(\lambda, E)\right|_{\lambda_{ \pm}}$. The two remaining integrals are known as a version of Selberg's integral [1], given by

$$
\int \prod_{i=1}^{p} \mathrm{~d} \xi_{i} \Delta_{p}^{2}\left(\hat{\Xi}_{-}\right) \exp \left\{-t \sum_{i=1}^{p} \xi_{i}^{2}\right\}=(2 t)^{-p^{2} / 2} \Omega_{p} \quad \text { with } \quad \Omega_{p}=(2 \pi)^{p / 2} \prod_{i=1}^{p} i !
$$

As a result the correlation function takes the form

$$
\begin{aligned}
S_{1}^{(n)}(E)=(i)^{1-n N} & \mathrm{e}^{-n N A_{+}} \sum_{p=0}^{n} F_{n}^{p}\left[\lambda_{+}-\lambda_{-}\right]^{2 p(n-p)} \frac{N^{p(n-p)}}{\left(\sqrt{A_{+}^{\prime \prime}}\right)^{(n-p)^{2}}\left(\sqrt{A_{-}^{\prime \prime}}\right)^{p^{2}}} \mathrm{e}^{p N\left(A_{+}-A_{-}\right)} \\
\times & {\left[p \lambda_{-}+(n-p) \lambda_{+}-\mathrm{i} n E\right] }
\end{aligned}
$$


where we have introduced the $F_{n}^{p}$ symbol as

$$
F_{n}^{p} \equiv C_{n}^{p} \frac{\prod_{j=1}^{p} j ! \prod_{j=1}^{n-p} j !}{\prod_{j=1}^{n} j !}=\prod_{j=1}^{p} \frac{\Gamma(j)}{\Gamma(n-j+1)} \quad p \neq 0
$$

and $F_{n}^{p=0}=1$. Since the $\Gamma$-function diverges at any negative integer, $F_{n}^{p>n}=0$. Therefore one may extend the summation over $p$ in equation (23) up to infinity. The resulting expression is suitable for the analytical continuation, $n \rightarrow 0$. To continue the $F_{n}^{p}$ function one may use the identity $\Gamma(-z)=-\pi /(\Gamma(z+1) \sin \pi z)$ with $z=j-1-n$, which leads to

$$
F_{n}^{p}=(-1)^{p(p-1) / 2}\left[\frac{\sin \pi n}{\pi}\right]^{p} \prod_{j=1}^{p} \Gamma(j) \Gamma(j-n) \quad p \neq 0 .
$$

Expanding the $\sin \pi n$ for small $n$, one obtains $F_{n \rightarrow 0}^{p=0}=1 ; F_{n \rightarrow 0}^{p=1}=n$, whereas $F_{n \rightarrow 0}^{p \geqslant 2}=\mathrm{O}\left(n^{p}\right)$. As a result only two terms with $p=0$ and $p=1$ survive in the sum in equation (23). This is an important conclusion: out of the $2^{n}$ possible saddle points only one with $p=0$ and $n$ with $p=1$ contribute in the replica limit. Of course, the argument we have given here is somewhat heuristic since the series in equation (23) is divergent for non-integer $n$. This should be justified more rigorously. Although we have not completed the rigorous proof of this statement, we present some elements of the proof in appendix B.

One may easily check that the $p=1$ contribution is smaller by a factor $1 / N$ with respect to the $p=0$ one. We are therefore back to the familiar statement that the large $N$ limit of the DOS may be calculated by the replica method using a single trivial saddle point for the $\hat{Q}$ matrix which is proportional to the unit matrix. In particular one obtains

$$
S_{1}(E)_{(p=0)}=\mathrm{i}\left(\lambda_{+}-\mathrm{i} E\right) .
$$

Employing equations (3) and (10) one finds the famous 'law of semicircle' for the DOS, $v_{0}(E)=\sqrt{4-E^{2}} /(2 \pi)$.

It is instructive to look at the $1 / N$ contribution originating from the $p=1$ saddle point manifold:

$$
S_{1}(E)_{(p=1)}=-\mathrm{i} \frac{1}{N} \frac{1}{\lambda_{+}-\lambda_{-}} \frac{\mathrm{e}^{N\left(A_{+}-A_{-}\right)}}{\sqrt{A_{+}^{\prime \prime} A_{-}^{\prime \prime}}} .
$$

This leads to an oscillatory correction to the mean DOS of the following form:

$$
\begin{aligned}
\delta v_{\text {osc }}(E)= & \frac{1}{N \pi} \frac{(-1)^{N+1}}{4-E^{2}} \cos [N(2 \theta+\sin 2 \theta)] \\
& =\frac{1}{N 4 \pi^{3}} \frac{1}{v_{0}^{2}(E)} \cos \left[2 \pi N \int_{-2}^{E} v_{0}(\tilde{E}) \mathrm{d} \tilde{E}+\pi\right]
\end{aligned}
$$

where $\sin \theta \equiv E / 2$. This is indeed the correct $1 / N$ oscillating correction, as can be checked directly using the exact, finite $N$, expression for the DOS [1]:

$$
\begin{gathered}
v(E)=\frac{\mathrm{e}^{-N E^{2} / 2}}{\sqrt{2 \pi N} 2^{N} \Gamma(N)}\left[H_{N}\left(E \sqrt{\frac{N}{2}}\right) H_{N}\left(E \sqrt{\frac{N}{2}}\right)\right. \\
\left.-H_{N-1}\left(E \sqrt{\frac{N}{2}}\right) H_{N+1}\left(E \sqrt{\frac{N}{2}}\right)\right]
\end{gathered}
$$

and employing the following integral representation of the Hermite polynomials:

$$
H_{N+k}\left(E \sqrt{\frac{N}{2}}\right)=\frac{(-2 \mathrm{i})^{N+k}}{\sqrt{\pi}}\left(\sqrt{\frac{N}{2}}\right)^{N+k+1} \int_{-\infty}^{\infty} \mathrm{d} \lambda \mathrm{e}^{-N A(\lambda, E)} \lambda^{k} .
$$


Evaluating this integral in the saddle point approximation, one obtains the 'semicircle' as the leading term and equation (28) as the $1 / N$ correction. Here we do not discuss the $1 / N$ correction to the smooth part of the DOS, which may be easily evaluated by expanding near the trivial $p=0$ saddle point $[9,16]$.

Notice that in deriving this result all $n+1$ saddle point manifolds in the $\hat{Q}$ space were taken into account. However, only two of them appear to contribute in the $n \rightarrow 0$ limit. In the leading order in $N \rightarrow \infty$ only the trivial one $\hat{Q} \sim \mathbb{1}$ remains, whereas the $p=1$ is responsible for the oscillatory $1 / N$ contribution to the DOS. We shall see below that in the case of the two-point correlation function non-trivial saddle point manifolds contribute already to the leading order.

These corrections due to the replica symmetry braking saddle point, which lead to an oscillatory behaviour of the density of states in the range $\lambda \in[-2,2]$, also lead to an exponentially small (in $N$ ) tail of the density of states outside of the interval $[-2,2]$. This fact was first noticed by Cavagna et al [17], who also showed that the $p=1$ saddle point reproduces the correct exponentially small tail.

\section{Two-point correlation function}

The two-point correlation function is defined as

$$
S_{2}\left(E, E^{\prime}\right)=N^{-2} \overline{\operatorname{Tr}(E-H)^{-1} \operatorname{Tr}\left(E^{\prime}-H\right)^{-1}}=N^{-2} \frac{\overline{\partial \ln Z(E)}}{\partial E} \frac{\partial \ln Z\left(E^{\prime}\right)}{\partial E^{\prime}}
$$

where complex energies $E$ and $E^{\prime}$ have negative and positive imaginary parts correspondingly. Introducing two sets of replicas with sizes $n$ and $n^{\prime}$, respectively, to handle each of the logarithms in this expression, one obtains

$$
S_{2}\left(E, E^{\prime}\right)=\lim _{n, n^{\prime} \rightarrow 0} \frac{1}{n n^{\prime}} S_{2}^{\left(n+n^{\prime}\right)}\left(E, E^{\prime}\right) \quad S_{2}^{\left(n+n^{\prime}\right)}=\frac{1}{N^{2}} \frac{\partial^{2}}{\partial E \partial E^{\prime}} \overline{Z(E)^{n} Z\left(E^{\prime}\right)^{n^{\prime}}} .
$$

We introduce again, for the sake of regularization, the function $Z^{\left(n+n^{\prime}\right)}(\hat{E})$, where $\hat{E}$ now, and in the rest of this section, is a diagonal $\left(n+n^{\prime}\right) \times\left(n+n^{\prime}\right)$ matrix of the form $\hat{E}=\operatorname{diag}\left\{E_{1}, \ldots, E_{n}, E_{n+1}, \ldots, E_{n+n^{\prime}}\right\} ;$ the limit $E_{1}, \ldots, E_{n} \rightarrow E ; E_{n+1}, \ldots, E_{n+n^{\prime}} \rightarrow E^{\prime}$ will be taken at the appropriate stage. The next steps are exactly identical to those of the previous section, up to the change of $n$ into $n+n^{\prime}$ : averaging over $H$, decoupling with the $\left(n+n^{\prime}\right) \times\left(n+n^{\prime}\right)$ matrix field $\hat{Q}$ and integrating over the group $U\left(n+n^{\prime}\right)$ using the ItzyksonZuber integral. This leads to the following result for the function $Z^{\left(n+n^{\prime}\right)}(\hat{E})$ (equivalent to that of equation (14)):

$$
\begin{aligned}
\overline{Z^{\left(n+n^{\prime}\right)}(\hat{E})}= & c_{n+n^{\prime}}(-\mathrm{i})^{N\left(n+n^{\prime}\right)}\left(\frac{\pi}{N}\right)^{\left(n+n^{\prime}\right)\left(n+n^{\prime}-1\right) / 2} \int \mathrm{d}[\hat{\Lambda}] \frac{\Delta_{n+n^{\prime}}(\hat{\Lambda})}{\Delta_{n+n^{\prime}}(\mathrm{i} \hat{E})} \\
& \times(\operatorname{det} \hat{\Lambda})^{N} \exp \left\{-\frac{N}{2} \sum_{j=1}^{n+n^{\prime}}\left(\lambda_{j}-\mathrm{i} E_{j}\right)^{2}\right\}
\end{aligned}
$$

where $\hat{\Lambda}$ is a diagonal $\left(n+n^{\prime}\right) \times\left(n+n^{\prime}\right)$ matrix containing the eigenvalues of the $\hat{Q}$ matrix. Once again, since the integral is a totally antisymmetric function of $E_{i}$, there are no poles at $E_{i}=E_{j}$. The next step is to take the limit $E_{j} \rightarrow E$ for $j=1, \ldots, n$ and $E_{j^{\prime}} \rightarrow E^{\prime}$ for $j^{\prime}=n+1, \ldots, n+n^{\prime}$. The corresponding limit is calculated in appendix A, the result is:

$\overline{Z^{n}(E) Z^{n^{\prime}}\left(E^{\prime}\right)}=c_{n}^{\prime} c_{n^{\prime}}^{\prime} \int \mathrm{d}[\hat{\Lambda}] \Delta_{n}^{2}\left(\hat{\Lambda}^{(n)}\right) \Delta_{n^{\prime}}^{2}\left(\hat{\Lambda}^{\left(n^{\prime}\right)}\right) \frac{\prod_{j=1}^{n} \prod_{j^{\prime}=n+1}^{n+n^{\prime}}\left(\lambda_{j^{\prime}}-\lambda_{j}\right)}{\left[\mathrm{i}\left(E^{\prime}-E\right)\right]^{n n^{\prime}}}$ 


$$
\times \exp \left\{-N \sum_{j=1}^{n} A\left(\lambda_{j}, E\right)-N \sum_{j^{\prime}=n+1}^{n+n^{\prime}} A\left(\lambda_{j^{\prime}}, E^{\prime}\right)\right\}
$$

where $\hat{\Lambda}^{(n)} \equiv \operatorname{diag}\left\{\lambda_{1}, \ldots, \lambda_{n}\right\}$ and $\hat{\Lambda}^{\left(n^{\prime}\right)} \equiv \operatorname{diag}\left\{\lambda_{n+1}, \ldots, \lambda_{n+n^{\prime}}\right\}$. Differentiation with respect to $E$ and $E^{\prime}$ gives the replicated two-point correlation function. Non-trivial correlations exist only in the range where $E-E^{\prime}$ is of the order of the level spacing, namely $1 / N$. We introduce thus scaling variables $\epsilon=N E$ and $\epsilon^{\prime}=N E^{\prime}$ and restrict ourselves to the vicinity of the center of the band $|\epsilon|,\left|\epsilon^{\prime}\right| \ll N$. The large $N$ limit of the correlation function at fixed $\epsilon, \epsilon^{\prime}$ is given by:

$$
\begin{aligned}
S_{2}^{\left(n+n^{\prime}\right)}\left(\epsilon, \epsilon^{\prime}\right)= & c_{n}^{\prime} c_{n^{\prime}}^{\prime} N^{n n^{\prime}} \int \mathrm{d}[\hat{\Lambda}] \Delta_{n}^{2}\left(\hat{\Lambda}^{(n)}\right) \Delta_{n^{\prime}}^{2}\left(\hat{\Lambda}^{\left(n^{\prime}\right)}\right) \frac{\prod_{j=1}^{n} \prod_{j^{\prime}=n+1}^{n+n^{\prime}}\left(\lambda_{j^{\prime}}-\lambda_{j}\right)}{\left[i\left(\epsilon^{\prime}-\epsilon\right)\right]^{n n^{\prime}}} \\
& \times \exp \left\{-N \sum_{j=1}^{n} A\left(\lambda_{j}, \frac{\epsilon}{N}\right)-N \sum_{j^{\prime}=n+1}^{n+n^{\prime}} A\left(\lambda_{j^{\prime}}, \frac{\epsilon^{\prime}}{N}\right)\right\} \\
& \times\left[-\sum_{j=1}^{n} \lambda_{j} \sum_{j^{\prime}=n+1}^{n+n^{\prime}} \lambda_{j^{\prime}}-\frac{i n n^{\prime}}{\epsilon^{\prime}-\epsilon}\left(\sum_{j=1}^{n} \lambda_{j}-\sum_{j^{\prime}=n+1}^{n+n^{\prime}} \lambda_{j^{\prime}}\right)-\frac{n n^{\prime}\left(1+n n^{\prime}\right)}{\left(\epsilon^{\prime}-\epsilon\right)^{2}}\right] .
\end{aligned}
$$

In the large $N$ limit the correlation function may be evaluated using the saddle point approximation. The saddle points of the integral in equation (35) are given by $\lambda_{j}= \pm 1$, for $j=1, \ldots n+n^{\prime}$, which is the zero energy limit of $\lambda_{ \pm}(E)$ discussed in the previous section. Altogether there are $2^{n+n^{\prime}}$ distinct saddle points. Each one of them may be parametrized in the following manner:

$$
\hat{\Lambda}=\operatorname{diag}\{\underbrace{-1, \ldots,-1}_{p}, \underbrace{+1, \ldots,+1}_{n-p}, \underbrace{+1, \ldots,+1}_{p^{\prime}}, \underbrace{-1, \ldots,-1}_{n^{\prime}-p^{\prime}}\}
$$

where $0 \leqslant p \leqslant n$ and $0 \leqslant p^{\prime} \leqslant n^{\prime}$. Given $p$ and $p^{\prime}$, there are $C_{n}^{p} C_{n^{\prime}}^{p^{\prime}}$ such saddle points. As before, one must be careful with the saddle point calculation because of the vanishing prefactors. The method we shall follow is the same as in the previous section. One introduces variables $\xi_{j}, \xi_{j^{\prime}}^{\prime}$, describing fluctuations around the saddle point:

$$
\begin{array}{ll}
\lambda_{i}=-1+\xi_{i} / \sqrt{N} & i=1 \ldots p \\
\lambda_{j}=+1+\xi_{j} / \sqrt{N} & j=p+1 \ldots n \\
\lambda_{i^{\prime}}=+1+\xi_{i^{\prime}}^{\prime} / \sqrt{N} & i^{\prime}=n+1 \ldots n+p^{\prime} \\
\lambda_{j^{\prime}}=-1+\xi_{j^{\prime}}^{\prime} / \sqrt{N} & j^{\prime}=n+p^{\prime}+1 \ldots n+n^{\prime}
\end{array}
$$

and groups them into diagonal matrices $\hat{\boldsymbol{\Xi}}_{-} \equiv \operatorname{diag}\left\{\xi_{1}, \ldots, \xi_{p}\right\}, \hat{\boldsymbol{\Xi}}_{+} \equiv \operatorname{diag}\left\{\xi_{p+1}, \ldots, \xi_{n}\right\}$, $\hat{\Xi}_{+}^{\prime} \equiv \operatorname{diag}\left\{\xi_{n+1}^{\prime}, \ldots, \xi_{n+p^{\prime}}^{\prime}\right\}, \hat{\Xi}_{-}^{\prime} \equiv \operatorname{diag}\left\{\xi_{n+p^{\prime}+1}^{\prime}, \ldots, \xi_{n+n^{\prime}}^{\prime}\right\}$. For large $N$ the determinants are decomposed as:

$$
\begin{array}{r}
\Delta_{n}\left(\hat{\Lambda}^{(n)}\right) \simeq\left(\frac{1}{\sqrt{N}}\right)^{p(p-1) / 2+(n-p)(n-p-1) / 2} 2^{p(n-p)} \Delta_{p}\left(\hat{\Xi}_{-}\right) \Delta_{n-p}\left(\hat{\Xi}_{+}\right) \\
\Delta_{n^{\prime}}\left(\hat{\Lambda}^{\left(n^{\prime}\right)}\right) \simeq\left(\frac{1}{\sqrt{N}}\right)^{p^{\prime}\left(p^{\prime}-1\right) / 2+\left(n^{\prime}-p^{\prime}\right)\left(n^{\prime}-p^{\prime}-1\right) / 2}(-2)^{p^{\prime}\left(n^{\prime}-p^{\prime}\right)} \Delta_{p^{\prime}}\left(\hat{\Xi}_{+}^{\prime}\right) \Delta_{n^{\prime}-p^{\prime}}\left(\hat{\Xi}_{-}^{\prime}\right) .
\end{array}
$$

Finally, the remaining factor in equation (35) takes the form

$$
\prod_{j=1}^{n} \prod_{j^{\prime}=n+1}^{n+n^{\prime}}\left(\lambda_{j^{\prime}}-\lambda_{j}\right) \simeq \frac{2^{p p^{\prime}}(-2)^{(n-p)\left(n^{\prime}-p^{\prime}\right)}}{(\sqrt{N})^{p\left(n^{\prime}-p^{\prime}\right)+(n-p) p^{\prime}}}\left[\prod_{i=1}^{p} \prod_{j^{\prime}=n+p^{\prime}+1}^{n+n^{\prime}}\left(\xi_{j^{\prime}}^{\prime}-\xi_{i}\right)\right]\left[\prod_{j=p+1}^{n} \prod_{i^{\prime}=n+1}^{n+p^{\prime}}\left(\xi_{i^{\prime}}^{\prime}-\xi_{j}\right)\right] \text {. }
$$


Expanding the exponent in equation (35) around the saddle point, equation (36),

$N A\left(\lambda_{j}, \frac{\epsilon}{N}\right) \simeq \frac{N}{2}-N \ln ( \pm 1) \mp \mathrm{i} \epsilon-\frac{\epsilon^{2}}{4 N}+\left(\xi_{j}-\frac{\mathrm{i} \epsilon}{2 \sqrt{N}}\right)^{2}+\mathrm{O}\left(\frac{1}{N^{3 / 2}}\right)$

one finds that the integrals over $\xi_{j}$ and $\xi_{j}^{\prime}$ may be expressed as $I_{p, n^{\prime}-p^{\prime}}\left(\mathrm{i}\left(\epsilon-\epsilon^{\prime}\right) / 2 \sqrt{N}\right) \times$ $I_{n-p, p^{\prime}}\left(\mathrm{i}\left(\epsilon-\epsilon^{\prime}\right) / 2 \sqrt{N}\right)$, where the function $I_{r, s}(a)$ is a generalization of Selberg's integral, defined as:

$I_{r, s}(a) \equiv \int \mathrm{d}[\hat{X}] \mathrm{d}[\hat{Y}] \Delta_{r}(\hat{X}) \Delta_{s}(\hat{Y}) \Delta_{r+s}(\hat{X} \oplus \hat{Y}) \exp \left\{-\sum_{j=1}^{r}\left(x_{j}-a\right)^{2}-\sum_{k=1}^{s} y_{k}^{2}\right\}$.

Here $\hat{X}, \hat{Y}$ and $\hat{X} \oplus \hat{Y}$ are diagonal matrices: $\hat{X}=\operatorname{diag}\left\{x_{1}, \ldots, x_{r}\right\}, \hat{Y}=\operatorname{diag}\left\{y_{1}, \ldots, y_{s}\right\}$, and $\hat{X} \oplus \hat{Y}=\operatorname{diag}\left\{x_{1}, \ldots, x_{r} y_{1}, \ldots, y_{s}\right\}$. We show in appendix $\mathrm{C}$ that this integral is given by

$$
I_{r, s}(a)=2^{-\left(r^{2}+s^{2}\right) / 2} \Omega_{r} \Omega_{s}(-a)^{r s}
$$

where $\Omega_{r}$ is the usual Selberg integral, equation (22). Up to an overall constant factor which goes to one in the limit $n, n^{\prime} \rightarrow 0$, one thus has:

$$
\begin{aligned}
C_{n}^{p} C_{n^{\prime}}^{p^{\prime}} I_{p, n^{\prime}-p^{\prime}} & \left(\frac{\mathrm{i}\left(\epsilon-\epsilon^{\prime}\right)}{2 \sqrt{N}}\right) I_{n-p, p^{\prime}}\left(\frac{\mathrm{i}\left(\epsilon-\epsilon^{\prime}\right)}{2 \sqrt{N}}\right) \\
= & F_{n}^{p} F_{n^{\prime}}^{p^{\prime}}\left(\frac{\mathrm{i}\left(\epsilon^{\prime}-\epsilon\right)}{\sqrt{N}}\right)^{p\left(n^{\prime}-p^{\prime}\right)+(n-p) p^{\prime}} 2^{-\left(p-p^{\prime}\right)^{2}} .
\end{aligned}
$$

Grouping all the terms, one finally obtains

$$
\begin{aligned}
S_{2}^{\left(n+n^{\prime}\right)}\left(\epsilon, \epsilon^{\prime}\right)= & \sum_{p=0}^{n} \sum_{p^{\prime}=0}^{n^{\prime}} F_{n}^{p} F_{n^{\prime}}^{p^{\prime}} \frac{(-1)^{p p^{\prime}} 2^{-3 p^{2}-3 p^{\prime 2}+4 p p^{\prime}} N^{\left(n-p+p^{\prime}\right)\left(n^{\prime}-p^{\prime}+p\right)}}{\left[\mathrm{i}\left(\epsilon^{\prime}-\epsilon\right)\right]^{n n^{\prime}-p\left(n^{\prime}-p^{\prime}\right)-(n-p) p^{\prime}}} \mathrm{e}^{2 \mathrm{i}\left(p^{\prime} \epsilon^{\prime}-p \epsilon\right)+\mathrm{i} \pi N\left(p-p^{\prime}\right)} \\
& \times\left[(n-2 p)\left(n^{\prime}-2 p^{\prime}\right)-\frac{\mathrm{i} n n^{\prime}}{\left(\epsilon^{\prime}-\epsilon\right)}\left(n-2 p+n^{\prime}-2 p^{\prime}\right)-\frac{n n^{\prime}\left(1+n n^{\prime}\right)}{\left(\epsilon^{\prime}-\epsilon\right)^{2}}\right]
\end{aligned}
$$

where we have omitted an inessential factor const ${ }^{\mathrm{O}(n)}$.

Employing the fact that $F_{n}^{p>n}=F_{n^{\prime}}^{p^{\prime}>n^{\prime}}=0$, one may extend summations over $p$ and $p^{\prime}$ to infinity and then perform the analytical continuation, $n, n^{\prime} \rightarrow 0$. Due to the properties of the $F$-symbol (cf equation (25)) only the terms with $p=0,1$ and $p^{\prime}=0,1$ contribute in the replica limit. We need to evaluate the contributions of these four saddle points. Recalling that $F_{n}^{0}=F_{n^{\prime}}^{0}=1$, one finds for the $p=p^{\prime}=0$ contribution to the two-point correlation function, $S_{2}\left(\epsilon, \epsilon^{\prime}\right)=\lim _{n, n^{\prime} \rightarrow 0}\left(n n^{\prime}\right)^{-1} S_{2}^{\left(n+n^{\prime}\right)}$ :

$$
S_{2}(\omega)_{\left(p=p^{\prime}=0\right)}=\left(1-\frac{1}{\omega^{2}}\right)
$$

where $\omega \equiv \epsilon-\epsilon^{\prime}$. This is the result obtained in the perturbation theory around the usual saddle point [18]. There is, however, an other contribution to $S_{2}$ originating from the saddle points with $p=p^{\prime}=1$. It is easily computed, using the fact that $F_{n}^{1}=n$ and $F_{n^{\prime}}^{1}=n^{\prime}$, and is equal to:

$$
S_{2}(\omega)_{\left(p=p^{\prime}=1\right)}=\frac{\mathrm{e}^{-2 \mathrm{i} \omega}}{\omega^{2}} .
$$

It is easy to check that the saddle points with $p=0, p^{\prime}=1$ and $p=1, p^{\prime}=0$ lead to $1 / N$ oscillatory correction to the disconnected part of the two-point correlation function, cf 
equation (28). Adding together the two leading terms, equations (45) and (46), one obtains the final result for the two-point correlation function:

$$
S_{2}(\omega)=1-\frac{1-\mathrm{e}^{-2 \mathrm{i} \omega}}{\omega^{2}}=1-2 \mathrm{i} \omega^{-2} \mathrm{e}^{-\mathrm{i} \omega} \sin \omega .
$$

Although this is the exact result $[1,4,8,10]$ for the GUE for any $\omega \ll N$, the way it was derived here only justifies it for $1 \ll \omega \ll N$. This is because of the terms which were omitted in the expansions (38) and (39). Although they look superficially as being of order $1 / \sqrt{N}$, they can, in fact, contribute. A careful examination shows that the generalized Selberg's integral is just the leading large $\omega(\omega \gg 1)$ contribution. We have thus computed only the leading term at large $\omega$ for each saddle point, but corrections could be incorporated systematically. Accidentally, the obtained expression appears to be exact down to zero $\omega$. A similar situation was already encountered in a SUSY approach, when the soft modes integrals were calculated with the saddle point method including an additional non-trivial saddle point [11]. In this sense our new saddle point with $p=p^{\prime}=1$ is a close analogue of the SUSY saddle point of Andreev and Altshuler [11].

\section{Discussion of the method}

Let us add some comments on the relation between our calculations and the saddle point evaluation of the $\sigma$-model, equation (8) with $\hat{E}=\operatorname{diag}\left\{E \mathbb{1}_{n}, E^{\prime} \mathbb{1}_{n^{\prime}}\right\}$. Looking for the saddle point solution in the form $\hat{Q}=U^{-1} \hat{\Lambda} U$, one finds the solution in the form

$$
\hat{Q}=\left(\begin{array}{cc}
V^{-1} & 0 \\
0 & V^{\prime-1}
\end{array}\right) \hat{\Lambda}\left(\begin{array}{cc}
V & 0 \\
0 & V^{\prime}
\end{array}\right)
$$

with $\hat{\Lambda}$ being a diagonal matrix, obeying $\Lambda^{2}+\mathrm{i} \hat{E} \hat{\Lambda}-\mathbb{1}=0$, and arbitrary $V \in U(n)$; $V^{\prime} \in U\left(n^{\prime}\right)$. For a diagonal matrix $\hat{\Lambda}$ of the structure given by equation (36), there is a set of rotations belonging to the coset space

$$
\frac{U(n)}{U(p) U(n-p)} \times \frac{U\left(n^{\prime}\right)}{U\left(p^{\prime}\right) U\left(n^{\prime}-p^{\prime}\right)}
$$

which leave the action invariant, while changing the saddle point matrix, $\hat{Q}$. As a result, there is a continuous saddle point manifold, which contains true zero modes of the functional integral, equation (8). In addition there are usual 'soft modes' with masses of order $\left|\epsilon-\epsilon^{\prime}\right| \ll N$. Notice that there are no zero modes around the trivial saddle point $p=p^{\prime}=0$. The saddle point, equation (36), contains $n-p+p^{\prime}$ components which are +1 and $n^{\prime}-p^{\prime}+p$ components which are -1 . Therefore, out of the total $\left(n+n^{\prime}\right)^{2}$ fluctuation directions $\left(n+n^{\prime}\right)^{2}-2\left(n-p+p^{\prime}\right)\left(n^{\prime}-p^{\prime}+p\right)$ are massive with the mass $N$, whereas the remaining $2\left(n-p+p^{\prime}\right)\left(n^{\prime}-p^{\prime}+p\right)$ degrees of freedom are split between $\left[n^{2}-p^{2}-(n-p)^{2}\right]+\left[n^{\prime 2}-p^{\prime 2}-\left(n^{\prime}-p^{\prime}\right)^{2}\right]=2\left(p(n-p)+p^{\prime}\left(n^{\prime}-p^{\prime}\right)\right)$ zero modes (cf (49)) and $2 n n^{\prime}-2 p\left(n^{\prime}-p^{\prime}\right)-2(n-p) p^{\prime}$ soft modes with the mass $\left|\epsilon-\epsilon^{\prime}\right| \ll N$. The integrals over the zero modes must be calculated exactly giving rise to the volume of the coset space (49). In the regime $1 \ll\left|\epsilon-\epsilon^{\prime}\right|$, the integrals over both massive and soft modes may be evaluated in the Gaussian approximation giving rise to factors $N^{-1 / 2}$ and $\left|\epsilon-\epsilon^{\prime}\right|^{-1 / 2}$ in the number of modes power. This is precisely the structure of equation (44), where the factor $F_{n}^{p} F_{n^{\prime}}^{p^{\prime}}$ is proportional to the volume of the coset space (49). The advantage of our method is an easy control over combinatorial factors, coefficients etc, otherwise it is equivalent to the Gaussian evaluation of the functional integral, equation (8), similar to that of [11] for the SUSY case.

Our method can be easily generalized for the higher-order correlation functions. For example, calculations of the three-point function $S_{3}\left(E, E^{\prime}, E^{\prime \prime}\right)$ with, say, $E$ having negative 
and $E^{\prime}, E^{\prime \prime}$ positive imaginary parts, lead to the triple sum over $p, p^{\prime}, p^{\prime \prime}$ analogous to that of equation (44). Again only the terms with $p, p^{\prime}, p^{\prime \prime}=0,1$ contribute in the replica limit. One may easily check that the correct result for the connected GUE three-point correlation function follows from the $p=p^{\prime}=1 ; p^{\prime \prime}=0$ and $p=p^{\prime \prime}=1 ; p^{\prime}=0$ terms, whereas all other possible combinations, including $p=p^{\prime}=p^{\prime \prime}=1$, appears to be small in powers of $1 / N$.

\section{Conclusion and perspectives}

There are several questions raised by our computation. An obvious one is to have a more rigorous derivation of the analytic continuation of the $g(x, n)$ function at small $n$ (see appendix B). Also, we have derived here only the asymptotic form of the correlation function at large energy differences, $\omega \gg 1$. We are convinced that the replica method allows to compute the correlation at all $\omega$, but an explicit construction would be interesting. Extending our approach to bosonic replicas and to other random matrix ensembles are among other open problems.

Here we have presented what we believe to be the first consistent application of the replica method to the RMT. Our computation reconciles the fermionic replica result with the previous approaches. The point of this paper is not to challenge these previous approaches. The results which we have derived here have been well known for years, and in fact there exist in the literature much stronger results on level spacing universality (see e.g. [15] and references therein). The $\sigma$-model representation itself has proven very successful when used with the SUSY method: in problems of random energy levels, the SUSY technique has been very well developed and has allowed one to derive many results in various problems of solid state and nuclear physics (see $[2,19]$ ). We think that our result has two interesting aspects: the mathematical consistency on the one hand, and the possibility to use these ideas for a study of disordered interacting electrons.

The previous situation in which the replica approach was considered as ill was not satisfactory from the mathematical point of view. Furthermore, the replica method is known to be highly successful in other problems such as the statistical mechanics of classical disordered systems (see [20] for a review) and localization theory [21], and its failure in the simple problem of eigenvalue correlations seemed strange. In this respect we would like to comment about the RSB which we have found. While the $\sigma$-model formulation seems to involve a $n \times n$ matrix order parameter, similar to the one which has been discussed for instance in spin glass problems, the symmetry groups are very different. In spin glasses the symmetry group of the replicated system is just the permutation group, while in the $\sigma$-model there is a larger symmetry group: in our case some version of the unitary group (depending on the type of correlation one computes, and whether one would use commuting or anti-commuting replicas). Integrating over the angular variables has left us with an order parameter (the set of $n$ eigenvalues of the $\hat{Q}$ matrix) which is a vector in the replica space. Therefore the pattern of the RSB which we have found is much more reminiscent of the 'vector RSB', discussed in the study of randomfield-like problems at low temperature [13], rather than the hierarchical RSB scheme, which describes the spin glass mean-field theory. The vector RSB may be traced back to the existence of several distinct ground state configurations in a problem. In all cases studied so far, there is an infinity of RSB saddle points, which contribute to the partition function. At the same time the SUSY approach cannot address these problems, because it is unable to estimate a sum over ground states, but rather computes a topological invariant, given by the sum over all saddle points, weighted by the parity of the number of unstable directions [22]. In the RMT the situation is much simpler: SUSY is exact, and there is only a finite number of saddle points contributing in the vector RSB (two saddle points in the DOS computation). These facts are 
certainly related, and it would be highly desirable to better understand their connection.

The SUSY method relies crucially on the fact that the original action (as in equation (5)) is quadratic in the field variables. In the application to electronic system, it is thus restricted to non-interacting electrons. The replica method does not have such a limitation, and it is therefore capable to address problems of interacting electrons [23]. It would be very interesting to see whether the new saddle points which we have found have some implications in the theory of interacting electrons in disordered media.

\section{Acknowledgments}

We greatly benefited from numerous discussions with A Andreev, I Gruzberg and S Nishigaki. We are grateful to G Parisi, C Tracy and M Zirnbauer for useful correspondence and for bringing some references to our attention. This research was supported in part by the National Science Foundation under grant No PHY94-07194. AK was supported by NSF grant DMR 93-0801.

\section{Appendix A.}

We first evaluate the integral given in equation (14), in the limit $E_{j} \rightarrow E, j \in\{1 \ldots n\}$. Denoting $E_{j}=E+\eta_{j}$, one rewrites the integral in the following form:

$\zeta\left(E_{1}, \ldots, E_{n}\right)=\int \mathrm{d}[\hat{\Lambda}] \Delta_{n}(\hat{\Lambda}) \exp \left\{-N \sum_{j=1}^{n} A\left(\lambda_{j}, E\right)+N \sum_{j=1}^{n} \lambda_{j}\left(\mathrm{i} \eta_{j}\right)-\frac{N}{2} \sum_{j=1}^{n} \eta_{j}^{2}\right\}$

where $A\left(\lambda_{j}, E\right)$ is defined by equation (16). Expanding the term $\exp \left\{N \sum_{j} \lambda_{j}\left(\mathrm{i} \eta_{j}\right)\right\}$ in series, one obtains:

$\zeta\left(E_{1}, \ldots, E_{n}\right)=\exp \left\{-\frac{N}{2} \sum_{j} \eta_{j}^{2}\right\} \sum_{k_{1}, \ldots, k_{n}=1}^{\infty} \frac{N^{k_{1}+\ldots k_{n}}}{k_{1} !, \ldots, k_{n} !}\left(\mathrm{i} \eta_{1}\right)^{k_{1}}, \ldots,\left(\mathrm{i} \eta_{n}\right)^{k_{n}} T_{k_{1}, \ldots, k_{n}}$

where the tensor $T$ is a function of $E$ defined as:

$$
T_{k_{1} \ldots k_{n}} \equiv \int \mathrm{d}[\hat{\Lambda}] \Delta_{n}(\hat{\Lambda}) \lambda_{1}^{k_{1}} \ldots \lambda_{n}^{k_{n}} \exp \left\{-N \sum_{j=1}^{n} A\left(\lambda_{j}, E\right)\right\} .
$$

Since the Vandermonde determinant is antisymmetric in $\lambda_{j}$, the tensor $T$ is fully antisymmetric: for any permutation $\pi$ of the $n$ indices, one has

$$
T_{k_{1} \ldots k_{n}}=T_{k_{\pi(1)} \ldots k_{\pi(n)}} S_{\pi}
$$

where $S_{\pi}= \pm 1$ is the signature of the permutation. In particular, one notices that $T_{k_{1}, \ldots, k_{n}}$ vanishes whenever two exponents $k_{i}$ and $k_{j}$ (with $i \neq j$ ) are equal. We are interested in the leading behaviour of $\zeta$ when all $\eta_{j}$ go to zero simultaneously. From the expression (A.2) and the antisymmetry of $T$, it is clear that the leading term is of order $\eta^{n(n-1) / 2}$ and is obtained whenever $k_{1}=0, k_{2}=1, \ldots, k_{n}=n-1$, or any permutation of the integers $0,1, \ldots n-1$, and the exponential prefactor may be neglected. The leading order in $\eta$ may be written as a sum over all permutations $\pi$ of the ensemble $\{0, \ldots, n-1\}$ :

$\zeta\left(E_{1}, \ldots, E_{n}\right) \simeq T_{01 \ldots n-1} N^{n(n-1) / 2} \frac{1}{\prod_{j=0}^{n-1} j !} \sum_{\pi} S_{\pi}\left(\mathrm{i} \eta_{1}\right)^{\pi(0)}, \ldots,\left(\mathrm{i} \eta_{n}\right)^{\pi(n-1)}$. 
In the sum over permutations one recognizes the Vandermonde determinant of the $i \eta_{j}$, which is equal to $\Delta_{n}(\mathrm{i} \hat{E})$ and thus cancels the corresponding factor in the denominator of equation (14). As for the value of $T_{01 \ldots n-1}$, it may be rewritten, again using the antisymmetry of $\Delta_{n}(\hat{\Lambda})$, as:

$$
\begin{aligned}
T_{01 \ldots n-1}= & \frac{1}{n !} \sum_{\pi} S_{\pi} \int \mathrm{d}[\hat{\Lambda}] \Delta_{n}(\hat{\Lambda}) \lambda_{1}^{\pi(0)} \ldots \lambda_{n}^{\pi(n-1)} \exp \left\{-N \sum_{j=1}^{n} A\left(\lambda_{j}, E\right)\right\} \\
= & \frac{1}{n !} \int \mathrm{d}[\hat{\Lambda}] \Delta_{n}^{2}(\hat{\Lambda}) \exp \left\{-N \sum_{j=1}^{n} A\left(\lambda_{j}, E\right)\right\} .
\end{aligned}
$$

Therefore, in the limit $\eta_{j} \rightarrow 0, \zeta$ behaves as:

$$
\zeta\left(E_{1}, \ldots, E_{n}\right) \simeq N^{n(n-1) / 2} \frac{1}{\prod_{j=0}^{n} j !} \Delta_{n}(\mathrm{i} \hat{E}) \int \mathrm{d}[\hat{\Lambda}] \Delta_{n}^{2}(\hat{\Lambda}) \exp \left\{-N \sum_{j=1}^{n} A\left(\lambda_{j}, E\right)\right\} .
$$

This establishes expression (15).

We now evaluate the $n+n^{\prime}$ dimensional integral appearing in equation (33), in the limit where $E_{j} \rightarrow E$ for $j=1 \ldots n$ and $E_{j^{\prime}} \rightarrow E^{\prime}$ for $j^{\prime}=n+1, \ldots, n+n^{\prime}$. The procedure is exactly the same as was explained above for the one-point function. We shall sketch it briefly. One writes $E_{j}=E+\eta_{j}$ and $E_{j^{\prime}}=E^{\prime}+\eta_{j^{\prime}-n}^{\prime}$ in terms of which the integral reads:

$$
\begin{aligned}
\zeta\left(E_{1}, \ldots, E_{n+n^{\prime}}\right) & =\int \mathrm{d}[\hat{\Lambda}] \Delta_{n+n^{\prime}}(\hat{\Lambda}) \exp \left\{-N \sum_{j=1}^{n} A\left(\lambda_{j}, E\right)-N \sum_{j^{\prime}=n+1}^{n+n^{\prime}} A\left(\lambda_{j^{\prime}}, E^{\prime}\right)\right\} \\
& \times \exp \left\{N \sum_{j=1}^{n} \lambda_{j}\left(\mathrm{i} \eta_{j}\right)+N \sum_{j^{\prime}=1}^{n^{\prime}} \lambda_{n+j^{\prime}}\left(\mathrm{i} \eta_{j^{\prime}}^{\prime}\right)-\frac{N}{2} \sum_{j=1}^{n} \eta_{j}^{2}-\frac{N}{2} \sum_{j^{\prime}=1}^{n^{\prime}} \eta_{j^{\prime}}^{\prime 2}\right\} .
\end{aligned}
$$

We expand the terms $\exp \left\{N \sum_{j} \lambda_{j}\left(\mathrm{i} \eta_{j}\right)\right\}$ and $\exp \left\{N \sum_{j^{\prime}} \lambda_{n+j^{\prime}}\left(\mathrm{i} \eta_{j^{\prime}}^{\prime}\right)\right\}$ in series, and notice that in the limit where all the $\eta_{j}$ and $\eta_{j^{\prime}}^{\prime}$ go to zero simultaneously and independently, the leading contribution, of order $(\eta)^{n(n-1) / 2}\left(\eta^{\prime}\right)^{n^{\prime}\left(n^{\prime}-1\right) / 2}$ is obtained when powers of $\mathrm{i} \eta_{j}\left(\right.$ resp. $\mathrm{i} \eta_{j}^{\prime}$ ) span the ensemble $\{0, \ldots, n-1\}$ (resp. $\left\{0, \ldots, n^{\prime}-1\right\}$ ). The result can be written as a sum over all permutations $\pi$ of the ensemble $\{0, \ldots, n-1\}$, and all permutations $\pi^{\prime}$ of the ensemble $\left\{0, \ldots, n^{\prime}-1\right\}$, in the following form:

$$
\begin{gathered}
\zeta\left(E_{1}, \ldots, E_{n+n^{\prime}}\right)=u N^{\frac{n(n-1)}{2}+\frac{n^{\prime}\left(n^{\prime}-1\right)}{2}}\left(\prod_{j=0}^{n-1} j ! \prod_{j^{\prime}=0}^{n^{\prime}-1} j^{\prime} !\right)^{-1} \sum_{\pi \pi^{\prime}} S_{\pi} S_{\pi^{\prime}}\left(\mathrm{i} \eta_{1}\right)^{\pi(0)} \ldots\left(\mathrm{i} \eta_{n}\right)^{\pi(n-1)} \\
\times\left(\mathrm{i} \eta_{1}^{\prime}\right)^{\pi^{\prime}(0)} \ldots\left(\mathrm{i} \eta_{n^{\prime}}^{\prime}\right)^{\pi^{\prime}\left(n^{\prime}-1\right)}
\end{gathered}
$$

where $u$ is equal to:

$$
\begin{aligned}
u=\int \mathrm{d}[\hat{\Lambda}] \Delta_{n+n^{\prime}}(\hat{\Lambda}) \lambda_{1}^{0} \lambda_{2}^{1} \ldots \lambda_{n}^{n-1} \lambda_{n+1}^{0} \lambda_{n+2}^{1} \ldots \lambda_{n+n^{\prime}}^{n^{\prime}-1} \\
\quad \times \exp \left\{-N \sum_{j=1}^{n} A\left(\lambda_{j}, E\right)-N \sum_{j^{\prime}=n+1}^{n+n^{\prime}} A\left(\lambda_{j^{\prime}}, E^{\prime}\right)\right\} .
\end{aligned}
$$

Exactly as above, this integral may be rewritten by permuting separately the dummy integration variables $\lambda_{1}, \ldots, \lambda_{n}$ and $\lambda_{n+1}, \ldots, \lambda_{n+n^{\prime}}$. One thus obtains in the limit where all $\eta_{j}, \eta_{j^{\prime}}^{\prime} \rightarrow 0$, in the notations of equation (34):

$$
\zeta\left(E_{1}, \ldots, E_{n+n^{\prime}}\right) \simeq N^{\frac{n(n-1)}{2}+\frac{n^{\prime}\left(n^{\prime}-1\right)}{2}}\left(\prod_{j=0}^{n} j ! \prod_{j^{\prime}=0}^{n^{\prime}} j^{\prime} !\right)^{-1} \prod_{1 \leqslant i<j \leqslant n}\left(E_{j}-E_{i}\right)
$$




$$
\begin{aligned}
& \times \prod_{n+1 \leqslant i^{\prime}<j^{\prime} \leqslant n+n^{\prime}}\left(E_{j^{\prime}}-E_{i^{\prime}}\right) \times \int \mathrm{d}[\hat{\Lambda}] \Delta_{n+n^{\prime}}(\hat{\Lambda}) \Delta_{n}\left(\hat{\Lambda}^{(n)}\right) \Delta_{n^{\prime}}\left(\hat{\Lambda}^{\left(n^{\prime}\right)}\right) \\
& \times \exp \left\{-N \sum_{j=1}^{n} A\left(\lambda_{j}, E\right)-N \sum_{j^{\prime}=n+1}^{n+n^{\prime}} A\left(\lambda_{j^{\prime}}, E^{\prime}\right)\right\} .
\end{aligned}
$$

This establishes expression (34).

\section{Appendix B.}

To prove the statement that only the terms with $p=0$ and $p=1$ contribute to the analytic continuation of equation (23) at small $n$, let us study the function $g(x, n)=\sum_{p=0}^{n} F_{n}^{p} x^{p}$. Using the Gaussian decomposition of the factors containing exponents of $p(n-p)$ and $p^{2}$, one may show that the correlation function $S_{1}^{(n)}$ is deduced from the knowledge of $g(x, n)$, where $x$ is a complex number, with a modulus slightly smaller than one $\dagger$. Using the fact that $F_{n}^{p+1}=F_{n}^{p} \Gamma(p+1) / \Gamma(n-p)$, one finds that for integer $n$ the function $g(x, n)$ satisfies the following integral equation:

$$
g(x, n)=1+x \int_{0}^{\infty} \mathrm{d} \tau \mathrm{e}^{-\tau} \int_{\mathcal{C}} \frac{\mathrm{d} u}{2 \pi} \frac{\mathrm{e}^{u}}{u^{n}} g(x \tau u, n)
$$

where $\mathcal{C}$ is the contour in the complex $u$-plane used for definition of the function $1 / \Gamma(z)$ : it goes around the negative real half axis, starting from $-\infty$ to zero with a small positive imaginary part, turning around zero and getting back to $-\infty$ with a small negative imaginary part, it thus passes around the cut of the $1 / u^{n}$ function for $n$ non-integer. This integral may be probably used to define the function $g(x, n)$ for an arbitrary $n$, although some further study of this statement is needed. Here we are interested in the behaviour of $g(x, n)$ at small $n$. Writing the first two terms in the small $n$ expansion as $g(x, n)=g_{0}(x)+n g_{1}(x)+\cdots$, one finds that $g_{0}$ and $g_{1}$ satisfy the following equations:

$$
\begin{aligned}
& g_{0}(x)=1+x \int_{0}^{\infty} \mathrm{d} \tau \mathrm{e}^{-\tau} \int_{\mathcal{C}} \frac{\mathrm{d} u}{2 \pi} \mathrm{e}^{u} g_{0}(x \tau u) \\
& g_{1}(x)=x \int_{0}^{\infty} \mathrm{d} \tau \mathrm{e}^{-\tau} \int_{\mathcal{C}} \frac{\mathrm{d} u}{2 \pi} \mathrm{e}^{u}\left[g_{1}(x \tau u)-g_{0}(x \tau u) \ln u\right] .
\end{aligned}
$$

Assuming that $g_{0}$ and $g_{1}$ are analytic in a certain domain $\mathcal{D}$ near the origin, one can compute them inside this domain by series expansion in powers of $x$. This leads immediately to $g_{0}=1$ and $g_{1}=x$, which gives exactly the same answer as our heuristic arguments given in the text. To complete the proof one has to find out a shape of $\mathcal{D}$. We believe that $\mathcal{D}$ is the part of the complex plane restricted by the unit circle, but we have not been able to prove it $\ddagger$.

\section{Appendix C.}

In this appendix we prove that the generalized Selberg integral, equation (41), is given by the expression (42). The proof consists of two steps:

$\dagger$ If one neglects an imaginary part of $E,|x|=1$. A small negative imaginary part of $E$ implies $|x|<1$. This is the reason for our choice of the parameterization of the saddle point, where there are $p$ eigenvalues $\lambda_{-}$, rather than the opposite choice where there would be $p$ eigenvalues $\lambda_{+}$.

$\ddagger$ One argument in favour of this is the fact that $g(x, n)$, for integer $n$, possesses the symmetry $g(x, n)=x^{n} g(1 / x, n)$; another indication comes from the fact that $|g(x, n)|$ is bounded at large $n$ for a fixed complex $x$ whenever $|x|<1$, while it diverges for $|x|>1$. 
(i) We shall prove that the series expansion of the integral in powers of $a$ starts as $a^{m}$ with $m \geqslant r s$. To this end we rewrite the integral as

$$
\begin{aligned}
I_{r, s}(a)=\int \mathrm{d}[\hat{X}] \mathrm{d}[\hat{Y}] \Delta_{r}(\hat{X}) \Delta_{s}(\hat{Y}) \Delta_{r+s}(\hat{X} \oplus \hat{Y}) \exp \left\{-\sum_{j=1}^{r} x_{j}^{2}-\sum_{k=1}^{s} y_{k}^{2}\right. \\
\left.+2 a \sum_{j=1}^{r} x_{j}-r a^{2}\right\}
\end{aligned}
$$

and notice that the integrand is equal to $\Delta_{r}(\hat{X})$ times a totally antisymmetric function of the $x_{j}$. This allows one to substitute in the integrand $\Delta_{r}(\hat{X})$ by $r ! x_{1}^{0} x_{2}^{1} \ldots x_{r}^{r-1}$. A similar observation for the $y$ variables allows one to substitute in the integrand $\Delta_{s}(\hat{Y})$ by $s ! y_{1}^{0} y_{2}^{1} \ldots y_{s}^{s-1}$, giving:

$$
\begin{aligned}
I_{r, s}(a)=r ! s ! \mathrm{e}^{-r a^{2}} \int \mathrm{d}[\hat{X}] \mathrm{d}[\hat{Y}] \Delta_{r+s}(\hat{X} \oplus \hat{Y})\left[x_{1}^{0} x_{2}^{1} \ldots x_{r}^{r-1} y_{1}^{0} y_{2}^{1} \ldots y_{s}^{s-1}\right] \\
\quad \times \exp \left\{-\sum_{j=1}^{r} x_{j}^{2}-\sum_{k=1}^{s} y_{k}^{2}+2 a \sum_{j=1}^{r} x_{j}\right\} .
\end{aligned}
$$

The integrand is the product of a term which is totally antisymmetric in all the $r+s$ integration variables times the factor $\left[x_{1}^{0} x_{2}^{1} \ldots x_{r}^{r-1} y_{1}^{0} y_{2}^{1} \ldots y_{s}^{s-1}\right] \exp \left\{2 a \sum_{j} x_{j}\right\}$. In this factor one can expand the exponential in a power series in $a$. Whenever there are two of the $r+s$ variables appearing with the same power, the integral is zero as can be seen by permuting these two variables. The first non-zero contribution appears, thus, when the power series generates a power like $y_{1}^{0} y_{2}^{1} \ldots y_{s}^{s-1} x_{1}^{\pi(s)} x_{2}^{\pi(s+1)} \ldots x_{r}^{\pi(s+r-1)}$, where $\pi$ is any permutation of the integers $s, s+1, \ldots, s+r-1$. Such terms appear when the exponential is expanded to the order $a^{r s}$. This shows that the series expansion of the integral in powers of $a$ starts at least with the order $a^{r s}$.

(ii) We demonstrate now that $I_{r, s}$ is a polynomial in $a$ of degree less or equal to $r s$. Shifting each $x_{j}$ to $x_{j}+a$ and splitting the factor $\Delta_{r+s}(\hat{X} \oplus \hat{Y})$, one may rewrite the integral as

$I_{r, s}(a)=\int \mathrm{d}[\hat{X}] \mathrm{d}[\hat{Y}] \Delta_{r}^{2}(\hat{X}) \Delta_{s}^{2}(\hat{Y}) \exp \left\{-\sum_{j=1}^{r} x_{j}^{2}-\sum_{k=1}^{s} y_{k}^{2}\right\} \prod_{j=1}^{r} \prod_{k=1}^{s}\left(y_{k}-x_{j}-a\right)$.

This shows that the integral is a polynomial in $a$ of a degree less or equal to $r s$.

As a result of (i) and (ii), $I_{r, s}(a)$ must be proportional to $a^{r s}$. Using equation (C.3), one finds that it may be expressed as a product of two usual Selberg integrals, equation (22), as:

$$
\begin{gathered}
I_{r, s}(a)=(-a)^{r s} \int \mathrm{d}[\hat{X}] \mathrm{d}[\hat{Y}] \Delta_{r}^{2}(\hat{X}) \Delta_{s}^{2}(\hat{Y}) \exp \left\{-\sum_{j=1}^{r} x_{j}^{2}-\sum_{k=1}^{s} y_{k}^{2}\right\} \\
=(-a)^{r s} 2^{-\left(r^{2}+s^{2}\right) / 2} \Omega_{r} \Omega_{s} .
\end{gathered}
$$

\section{References}

[1] Mehta M L 1991 Random Matrices 2nd edn (Boston, MA: Academic)

[2] Guhr T, Mueller-Groeling A and Weidenmueller H A 1998 Phys. Rep. 299189 and references therein

[3] Gaudin M 1961 Nucl. Phys. 25447

[4] Dyson F J 1962 J. Math. Phys. 3140

Dyson F J 1962 J. Math. Phys. 3157

Dyson F J 1962 J. Math. Phys. 3166

Dyson F J 1962 J. Math. Phys. 31199

[5] Wegner F K 1979 Z. Phys. B 35207

[6] Efetov K B, Larkin A I and Khmelnitskii D E 1980 Zh. Eksp. Teor. Fiz. 791120 
Efetov K B, Larkin A I and Khmelnitskii D E 1980 Sov. Phys.-JETP 52568

[7] Khmelnitskii D E Private communication (see also remarks in [10])

[8] Verbaarschot J J M and Zirnbauer M R 1985 J. Phys. A: Math. Gen. 171093

[9] Itoi C, Mukaida H and Sakamoto Y 1997 J. Phys. A: Math. Gen. 305709

[10] Efetov K B 1983 Adv. Phys. 3253

[11] Andreev A V and Altshuler B L 1995 Phys. Rev. Lett. 75902

[12] Itzykson C and Zuber J B 1980 J. Math. Phys. 198021411

[13] Dotsenko V and Mézard M 1997 J. Phys. A: Math. Gen. 303363

[14] Guhr T 1991 J. Math. Phys. 32336

[15] Brézin E and Hikami S 1997 Phys. Rev. E 56264

[16] Verbaarschot J J M and Zirnbauer M R 1984 Ann. Phys., Lpz. 15878

[17] Cavagna A, Giardina I and Parisi G 1998 Phys. Rev. B 5711251

[18] Altshuler B L and Shklovskii B I 1986 Zh. Eksp. Teor. Fiz. 91220 Altshuler B L and Shklovskii B I 1986 Sov. Phys.-JETP 64127

[19] Efetov K B 1997 Supersymmetry in Disorder and Chaos (Cambridge: Cambridge University Press)

[20] Mézard M, Parisi G and Virasoro M A 1987 Spin Glass Theory and Beyond (Singapore: World Scientific)

[21] Gruzberg I A and Mirlin A D 1996 J. Phys. A: Math. Gen. 295333

[22] Parisi G 1984 Proceedings of Les Houches 1982 ed J B Zuber and R Stora (Amsterdam: North Holland)

[23] Finkel'stein A M 1983 Zh. Eksp. Teor. Fiz. 84168

Finkel'stein A M 1983 Sov. Phys.-JETP 5797 